\section{Análisis temporal de la sequía meteorológica en localidades semiáridas de Venezuela*}

\section{Temporal analysis of meteorological drought in semi-arid areas of Venezuela}

\author{
Barlin Orlando Olivares**, Adriana Cortez***, María F. Rodríguez***, Raquel Mayela Pa- \\ rra****, Deyanira Lobo*****, Juan C. Rey B***,*****
} * Proyecto de investigación: Fortalecimiento de la Red Agrometeorológica del Instituto Nacional de Investigaciones
Agrícolas (INIA). Acción: Generación de información agrometeorológica de la red de estaciones a nivel nacional. Institución:
INIA-Gerencia de Investigación e Innovación Tecnológica. Sede: Centro Nacional de Investigaciones Agropecuarias
(CENIAP). Maracay estado Aragua, Venezuela.

**Investigador. Doctorando del Programa Iberoamericano de Doctores en Agroalimentación de la Universidad de Córdoba (UCO), España. Correo:barlinolivares@gmail.com

***INIA. Centro Nacional de Investigaciones Agropecuarias (CENIAP). Laboratorio de sistemas de Información en Recursos Agroecológicos, Aragua, Venezuela.

****Universidad Central de Venezuela (UCV). Facultad de Agronomía, Departamento de Ingeniería Agrícola, Aragua, Venezuela.

*****Universidad Central de Venezuela (UCV). Facultad de Agronomía, Departamento de Edafología, Aragua, Venezuela.

\section{Resumen}

La sequía es uno de los temas de investigación ambiental de gran importancia en el mundo, principalmente en los países en vías de desarrollo con zonas áridas y semiáridas. El objetivo de esta investigación fue caracterizar la sequía meteorológica en una de las regiones naturales más secas de Venezuela, tomando como base la información de precipitación de cuatro estaciones de la red agrometeorológica del Instituto Nacional de Investigaciones Agrícolas (INIA) ubicadas en el estado Lara y Yaracuy, mediante el Índice de Precipitación Estandarizado (SPI), el cual permitió identificar y describir este fenómeno, precisando su inicio, intensidad, duración, magnitud y frecuencia de ocurrencia. De acuerdo con los valores de SPI reportados existe el fenómeno de la sequía meteorológica, mostrando variaciones temporales de la intensidad y magnitud. Las magnitudes máximas más relevantes fueron las registradas en Quíbor en el 2001 con 11,29 y El Cují durante el 2005 con un valor de 11,18. Los resultados reportados en este estudio servirán de base de información en las labores de planificación dirigidas en el Plan Nacional de Ordenación del Territorio, orientadas a definir e implementar estrategias, convirtiéndolas en el elemento central del proceso de desarrollo, logrando así una contribución a la reducción de la vulnerabilidad de las comunidades agrícolas de esta región.

Palabras clave: Cambio climático, déficit hídrico, precipitación, región semiárida.
Recibido: $12 / 02 / 2016$

Revisado: 25/03/2016

Aceptado: 10/12/2016

Correspondencia de autor: barlinolivares@gmail.com

C 2016 Universidad La Gran Colombia. Este es un artículo de acceso abierto, distribuido bajo los términos de la licencia Creative Commons Attribution License, que permite el uso ilimitado, distribución y reproducción en cualquier medio, siempre que el autor original y la fuente se acrediten.

Cómo citar:

Olivares,B.O., Cortez,A., Parra, R., Lobo, D., Rey, J., Rodriguez, M. (2016) Análisis temporal de la sequía meteorológica en localidades semiáridas de venezuelaUGCiencia 22, 11-24.

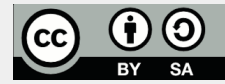




\begin{abstract}
Drought is one of the most important environmental research topics of the world, mainly in developing countries with arid and semi-arid areas. The purpose of this research was to characterize meteorological drought in one of the driest natural regions of Venezuela, using rainfall information of four seasons of the agro-meteorological network of the National Institute of Agricultural Research (NIAR), located in the States of Lara and Yaracuy, through the Standardized Rainfall Index (SRI), which led to identify and describe this phenomenon, establishing its initiation, intensity, duration, magnitude and frequency. According to reported SPI figures, the phenomenon of meteorological drought shows temporal variations of intensity and magnitude. The most important maximum magnitudes were those registered in Quibor, in 2001 with 11,29 and El Cuji in 2005, with a figure of 11,18. Results reported herein will serve as information basis for planning guided by the National Plan Organization of the Territory, aimed at defining and implement strategies, turning them into the central element of the development process, thus contributing to reduce vulnerability of agricultural communities of this region.
\end{abstract}

Key words: Climatic change, hydric deficit, rainfall, semi-arid region.

\section{Introducción}

La sequía es considerada por expertos como uno de los eventos climáticos más complejos y poco comprendido a nivel mundial, debido principalmente a su característica de extenderse de forma irregular en el tiempo y espacio, a diferencia de otros desastres naturales (Wilhite, 2000; Nuñez, 2013; Ortega-Gaucin y Velasco, 2013). Es precisamente por esta razón que resulta difícil proporcionarle un enfoque genérico que contemple todos los aspectos $\mathrm{y}$ satisfaga todas las expectativas, por lo que la sequía responde más bien a una particularidad del clima y del medio ambiente, lo que la convierte en un fenómeno con carácter relativo y elusivo (Velasco Ochoa y Gutiérrez, 2005).

En Venezuela, son recientes los estudios detallados sobre la ocurrencia y dinámica de las sequías meteorológica, agrícola e hidrológica. Dentro de los estudios más destacados en este tema se encuentran los aportes de Guevara y Paredes (2007); Paredes Millano y Guevara (2008); Paredes y Guevara (2010). Entre los estudios encontrados con relación a la seguía agrícola están los realizados por Ibarra y
Mendoza (2004); Mendoza, Méndez y Lobo (2007); Maracara (2007); Mendoza y Puche (2007). Por su parte, la sequía hidrológica ha sido estudiada por Paredes, Rumbo, Guevara y Carballo (2006) y Hernández (2008).

Los estudios citados han abordado el problema de la sequía en diferentes zonas del país, siendo el occidente, especialmente la región semiárida denominada sistema coriano, la zona donde existe menos énfasis en los estudios de sequía en el país. De allí, la importancia de establecer el análisis de este fenómeno meteorológico en esta zona esencialmente por las destacadas actividades económicas en el ámbito comercial, la financiera, industrial, agroindustrial y agrícola, relacionada con los rubros de café, papa y horticultura de pisos altos en las áreas más húmedas y caña de azúcar y hortalizas de piso bajo con riego en las áreas secas, así como, los cultivos de piña, sisal; ganadería de doble propósito y la ganadería ovina y caprina extensiva constituye la principal explotación para el sustento de los habitantes de las zonas secas (Marn, 2004). 
Por otra parte, se plantea entonces el problema asociado a que en Venezuela existe información en un nivel muy general sobre las áreas más propensas a sufrir desertificación, pero casi no existe información cuantificada, por medio de índices, sobre el riesgo de ocurrencia, tipo o intensidad de las sequías a nivel de mesoescala, información que resulta valiosa y significativa que servirá de base para comprender en un sentido más amplio el problema de la desertificación y sus relaciones con la sequía.

El progreso en las investigaciones representadas por el estudio del fenómeno de sequía: causas, consecuencias, estrategias de evaluación y mitigación, entre otros temas, resultan insuficientes para encontrar los mecanismos adecuados para prevenir, planear y mitigar los efectos de esta en la nación. El análisis precedente, refleja que aunque existen instituciones nacionales y regionales, universidades e instituciones de investigación que abordan el estudio de este fenómeno, es evidente que el avance es desigual y en muchos lugares incipiente o prácticamente nulo. Comúnmente se subestima la planeación de estrategias de evaluación en regiones donde la sequía no se presenta con frecuencia, no se cuenta con recursos financieros suficientes, tanto para investigar como para atender los efectos del fenómeno, y en ocasiones no existe aún una política de manejo y conservación de recursos naturales, incluyendo el agua.

Por las consideraciones expuestas y con la finalidad de confrontar el fenómeno de las sequías meteorológicas en localidades de importancia agrícola ubicadas en la región natural denominada Sistema Coriano, surge la necesidad de caracterizar este fenómeno mediante el cálculo del Índice de Precipitación Estandarizado (en inglés SPI, Standardized Precipitation Index), obteniendo resultados que coadyuvarán a la identificación de las áreas vulnerables a la sequía en la regiones en estudio, donde se encuentran las mayores superficies de cultivos de secano en el país, a partir de la cual se podrán establecer propuestas para el diseño de estrategias orientadas al manejo integral del recurso hídrico y que contribuyan a reducir el riesgo para la seguridad agroalimentaria.

\section{Materiales y métodos}

\section{Unidad de estudio}

En esta investigación la unidad de estudio está constituida por las cuatro estaciones (Yaritagua, Quíbor, El Cují y Las Cuibas) que conforman la red de estaciones agrometeorológicas del INIA, abarcando la región natural del sistema Coriano (estados: Lara y Yaracuy) (figura 1). Es una región de relieve variado y poco elevado, con altitudes entre 500 y $1.700 \mathrm{msnm}$. Se ubica al noroeste del país y ocupa la casi totalidad de los estados Falcón, Lara y Yaracuy, con un área aproximada de $52.000 \mathrm{~km}^{2}$.

Existe un marcado déficit hídrico, con extremos secos y húmedos y zonas de transición climática; en sentido general se observan dos tipos de clima: uno seco o semiárido, de escasa precipitación y alta evapotranspiración, que domina en la parte central y norte correspondiente a la depresión de Carora-Quíbor-Barquisimeto, y la de Baragua-Siquisique, con serranías a sus alrededores e interiores y otro subhúmedo a húmedo al sur y sureste del estado. (Marn, 2004; 2005). 
Figura 1. Distribución geográfica de la red de estaciones agrometeorológicas del INIA en la región natural del Sistema Coriano en Venezuela. Fuente: Elaboración propia.

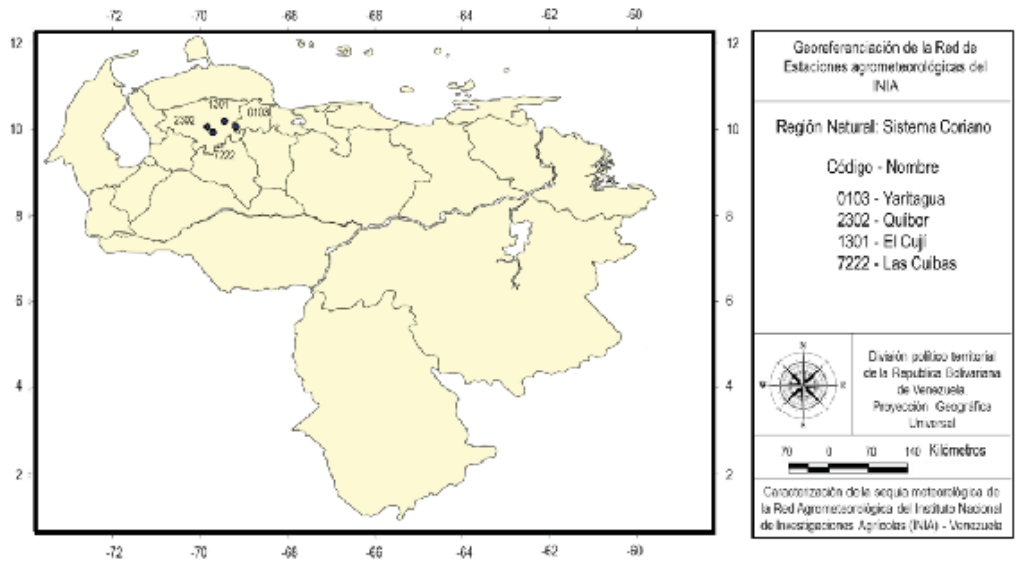

La tabla 1 presenta la información de la metadata básica representada por el serial nacional, el cual es un código asignado por el organismo responsable de la estación meteorológica, nombre de la estación, fecha de instalación, altitud en msnm, ubicación en grados, minutos y segundos para obtener la georeferenciación (latitud y longitud). Es conveniente señalar que el periodo de años utilizados en este estudio corresponde al indicado desde la fecha de instalación hasta el último año de registro de cada estación agrometeorológica con la finalidad de conocer la variación temporal de los eventos de sequía en el trascurso histórico para esas localidades.

Cuadro 1. Listado de estaciones agrometeorológicas bajo estudio

\begin{tabular}{lcccccc}
\hline Estación & Estado & Serial & Periodo & $\begin{array}{c}\text { Altura } \\
\text { m.s.n.m }\end{array}$ & $\begin{array}{c}\text { Latitud } \\
\text { dec. }\end{array}$ & $\begin{array}{c}\text { Longitud } \\
\text { dec. }\end{array}$ \\
\hline El Cuji & Lara & 1301 & $1980-2014$ & 605 & 10,0851 & $-69,1853$ \\
Quibor & Lara & 2302 & $1980-2014$ & 705 & 9,5314 & $-69,3911$ \\
Las Cuibas & Lara & 7222 & $2004-2014$ & 1648 & 9,484 & $-69,3235$ \\
Yaritagua & Yaracuy & 103 & $1980-2014$ & 308 & 10,0238 & $-69,0520$ \\
\hline \multicolumn{7}{c}{ Fuente: Elaboración propia. }
\end{tabular}

Procedimientos aplicados para el control de calidad de datos climáticos

Se aplicó el control de calidad a las series de precipitación diaria de las estaciones del INIA, mediante la determinación de datos faltantes, el cálculo de estadística descriptiva, análisis de concentración y tendencia de series temporales y análisis de dispersión de acuerdo con la metodología propuesta por Parra y Cortez (2005); y los procedimientos estadísticos desarrollados por Ablan. Andressen, Vargas y Acevedo (2008) y Olivares, Cortez, Parra, Rodríguez y Guevara (2013) en estaciones climatológicas a nivel nacional. 
La tabla 2 presenta un resumen de los procedimientos estadísticos aplicados a las series de precipitación, el principal problema característico lo constituyen los datos faltantes mensuales, afectando la longitud de las series que se quieren analizar. El resto de los procedimientos estadísticos arrojó resultados adecuados dentro de lo esperado.

Tabla 2. Resultados de la aplicación de los prcedimientos para el control de calidad de las series de precipitación de las estaciones agrometeorológicas estudiadas durante el periodo histórico

\begin{tabular}{lccccc}
\hline Estación & $\begin{array}{c}\text { Datos } \\
\text { Faltantes } \\
\text { Mensuales } \\
(\%)\end{array}$ & $\begin{array}{c}\text { Datos } \\
\text { Extremos }\end{array}$ & $\begin{array}{c}\text { Estadistica } \\
\text { Descriptiva }\end{array}$ & $\begin{array}{c}\mathbf{N}^{\circ} \text { de series } \\
\text { aleatorias }\end{array}$ & $\begin{array}{c}\text { Análisis de } \\
\text { dispersión }\end{array}$ \\
\hline El Cuji & 47 & No & N & 10 & $\mathrm{~N}$ \\
Quibor & 77 & No & N & 11 & N \\
Las Cuibas & 3 & No & N & 10 & N \\
Yaritagua & 18 & No & N & 9 & N \\
\hline
\end{tabular}

Nota: (N: Patrón Normal esperado).

Fuente: Elaboración propia.

\section{Determinación del Î́ndice de Precipitación}

\section{Estandarizado (SPI)}

En este trabajo, para la caracterización de la sequía en las áreas bajo estudio, se utilizó el Índice de Precipitación Estandarizado (SPI), desarrollado por Mckee, Doesken y Kleist (1993). Para lo cual se requiere únicamente el uso de series históricas de precipitación mensual y ofrece la ventaja de manipular diversas escalas de tiempo, haciendo posible identificar los impactos de la sequía en periodos de corto, mediano y largo plazo; además permite identificar y describir convenientemente el fenómeno, precisando su inicio, intensidad, duración, magnitud y cobertura espacial. Este método admite que la lluvia es el principal factor que define si un período o área determinados son o no deficientes de agua.
Para cada mes se determinó el valor acumulado mensual, obteniéndose así 12 sub-series en cada estación. Se estimaron los parámetros $\alpha$ y $ß$ de la Distribución de Probabilidad Teórica Gamma (DPTG) asociada a cada una de esas 12 sub-series de precipitación acumulada. La función de densidad de probabilidad de la DPTG está dada por la ecuación 1:

$$
f(x, \alpha, \beta)=\frac{1}{\beta^{\alpha} \Gamma(\alpha)} x^{\alpha-1} e^{-x / \beta}
$$

Donde $f(\mathrm{x}, \alpha, \beta)$ : es la función de densidad de probabilidad Gamma, (x): es la lluvia acumulada mensual expresada en $\mathrm{mm} ;(\alpha$ y $\beta)$ : son los parámetros de escala y de forma de la distribución, respectivamente y $\Gamma$ : Distribución Gamma. Por otra parte, la probabilidad de que el acumulado mensual, en una subserie, sea menor o igual al registro existente, se representa como $F(x)$ y se estima según la ecuación 2 :

$$
F(x)=\int_{0}^{x} f(x, \alpha, \beta) d x
$$

Para estimar los parámetros $\alpha$ y $\beta$ de la ecuación 1, se utilizó la metodología propuesta por Campos (2005), que se resume a continuación: en primer lugar, se calcula para cada subserie mensual, una variable auxiliar adimensional (A), definida de la siguiente manera

Donde $\ln \left(\mathrm{x}_{\mathrm{i}}\right)$ es el logaritmo neperiano del

$$
\mathrm{A}=\ln (\overline{\mathrm{x}})-\frac{\sum_{1}^{\mathrm{n}^{\prime}} \ln \left(\mathrm{x}_{\mathrm{i}}\right)}{\mathrm{n}^{\prime}}
$$

registro acumulado, $\mathrm{n}^{\prime}$ representa el número de registros no nulos, y $\bar{X}$ es la media aritmética de la subserie mensual expresada en $\mathrm{mm}$. Del paso anterior se obtienen 12 variables auxiliares, A. Posteriormente, la estimación 
de los parámetros $\alpha$ y $\beta$ de cada subserie se realiza aplicando las ecuaciones 4 y 5 .

$$
\begin{gathered}
\alpha=\frac{1+\sqrt{1+\frac{4}{3}} \mathrm{~A}}{4 \mathrm{~A}} \\
\beta=\frac{\bar{X}}{\alpha}
\end{gathered}
$$

Los registros nulos en las subseries mensuales imposibilitan calcular la variable auxiliar A (el logaritmo neperiano de cero tiende a infinito), por lo tanto, se empleó la Función Gamma Mixta (FGM) propuesta por Thom (1971) y Wu, Hayes, Wilhite y Svoboda (2005) como sigue en la ecuación 6 :

$$
\mathrm{H}(\mathrm{x})=\mathrm{q}+\mathrm{p} F(\mathrm{x})
$$

Donde (q) es la probabilidad de que se presente un valor nulo en la sub-serie, $(p=1-q)$ es la probabilidad de que no se presente un valor nulo en la sub-serie, y $\mathrm{H}(\mathrm{x})$ es la probabilidad de no excedencia del registro. Una vez obtenidas las 12 series de probabilidades Gamma, se estimó el valor $\mathrm{Z}$ o valor de SPI que le corresponde, en una distribución normal estandarizada con media cero y desviación estándar igual a 1 .

\section{Categorización de la intensidad del SPI}

McKee, Doesken y Kleist (1993) utilizaron el sistema de clasificación mostrado en la tabla de valores de SPI que se muestra en la tabla 3 , para definir las distintas intensidades de la sequía según los distintos valores de SPI. Estas categorías están referidas al fenómeno de la sequía meteorológica (aquellas cuyo valor de SPI es negativo) y por tanto, corresponden a eventos secos coyunturales, mas no constituyen una condición de aridez (Colotti, Cedeño y Montañez, 2013).
Tabla 3. Clasificación del SPI (Fuente: McKee, Doesken y Kleist, 1993)

\begin{tabular}{ll}
\hline SPI & Categoría \\
\hline 2,0 y más & extremadamente húmedo \\
1,5 a 1,99 & muy húmedo \\
1,0 a 1,49 & moderadamente húmedo \\
$-0,99$ a 0,99 & normal o aproximadamente normal \\
$-1,0$ a $-1,49$ & moderadamente seco \\
$-1,5$ a $-1,99$ & severamente seco \\
-2 y menos & extremadamente seco \\
\hline
\end{tabular}

\section{Determinación de la magnitud, duración y frecuencia de ocurrencia de la sequía}

Para calcular la magnitud del periodo seco durante un año cualquiera, en una estación determinada, se empleó una variante del método original propuesto por Edwards y Mckee (1997) donde se acumularon los SPI mensuales cuya magnitud era igual o inferior a -1 , y cuando el SPI era mayor a -1 se sustituyó por un cero. Bajo este enfoque, un valor de SPI >-1 indica una condición normal o húmeda (ecuación 7)

$$
\mathrm{MS}=-\sum_{\mathrm{I}=1}^{12} \mathrm{SPI}_{\mathrm{i}} \quad \text { Sí y sólo sí } \mathrm{SPI}_{\mathrm{i}}<0
$$

Donde (MS): representa la magnitud de la sequía para el período evaluado, (SPI): es el índice SPI para series de lluvia acumulada mensual. La tabla 4 muestra las categorías de la magnitud de la sequía.

Tabla 4. Magnitud de la sequía (Fuente: Hernández, 2008)

\begin{tabular}{ll}
\hline MS & Categoría \\
\hline $0,1-0,9$ & Normal \\
$1-1,99$ & Leve \\
$2-2,99$ & Poco fuerte \\
$3-3,99$ & Fuerte \\
$4-4,99$ & Muy fuerte \\
$>5$ & Extremadamente fuerte \\
\hline
\end{tabular}


Según McKee, Doesken y Kleist (1993) los valores del SPI continuamente negativos alcanzando el valor (-1) o inferior, son considerados una secuencia seca significativa relacionada con la deficiencia suficientemente importante de agua, mientras que los valores positivos se identifican con la categoría normal o húmeda. La frecuencia de ocurrencia se determinó mediante el número de casos de sequía que se producen durante un periodo determinado, estableciendo así, la probabilidad empírica de que ocurra una sequía de determinada magnitud. Para la realización de todos los cálculos se utilizó una hoja de cálculo de Microsoft Office Excel 2013.

\section{Resultados}

\section{Intensidad de la sequía}

La figura 2 muestra la intensidad de la sequía en la estación El Cují. De acuerdo con la precipitación registrada en esta zona se presentaron valores de sequía extrema en el $1,59 \%$, de los años, mientras que la intensidad severa alcanzó el $2,39 \%$. Nuevamente, la intensidad moderada predomina en esta estación con un $8,22 \%$ del total de SPI mensual.

Figura 2. Transcurso del SPI mensual para el periodo (1980-2014) en la estación El Cují.

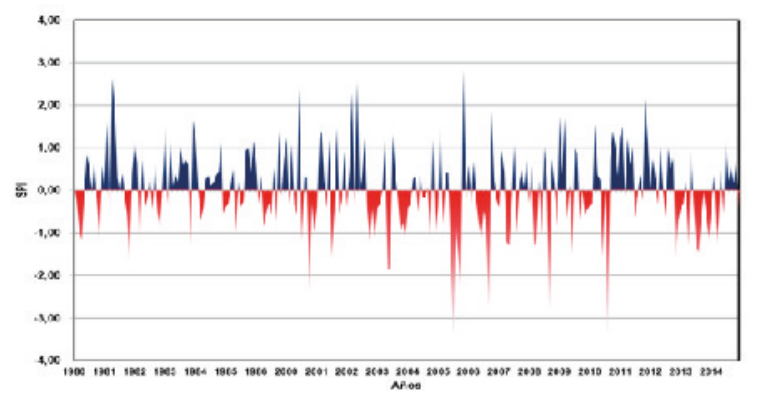

Fuente: Elaboración propia.
En la estación Quíbor, los valores de sequía extrema representan el $1,34 \%$ del total de SPI mensuales, la intensidad severa alcanzó un $2,68 \%$ y la moderada fue de un $9,38 \%$ (figura 3). Tal y como se observa, el año 2001 presentó una sequía que inició en abril y finalizó en agosto. En estas zonas subhúmedas secas, el agua disponible en condiciones normales es apenas suficiente para mantener las actividades agrícolas de subsistencia, por ende, cualquier disminución, incluso tan pequeña que no pueda considerarse como una sequía, tiene impactos negativos, que serán sumamente graves a largo plazo.

Figura 3. Transcurso del SPI mensual para el periodo (1980-2012) en la estación Quíbor.

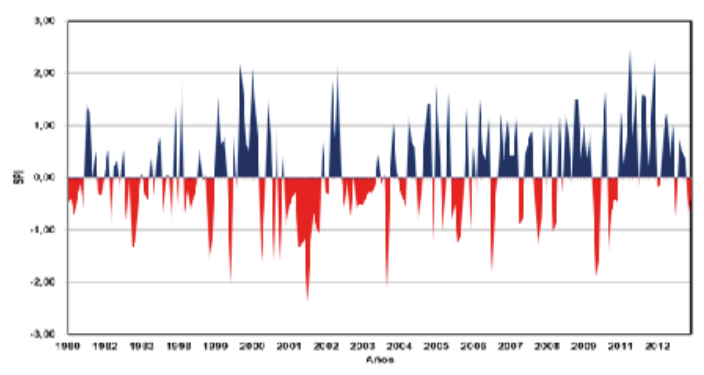

Fuente: Elaboración propia.

El transcurso del SPI mensual para el periodo evaluado en la estación Yaritagua, se ve reflejado en la figura 4; predominando la intensidad moderada con un $5,75 \%$ del total de SPI mensuales. Los valores de sequía extrema representan el $2,05 \%$ y la intensidad severa el 3,49\%. Por su parte la estación Las Cuibas (figura 5) presentó valores de sequía extrema en el 1,52\%, de los años, mientras que la intensidad severa alcanzó el 3,79\%. De acuerdo con los datos obtenidos existe evidencia de sequías con una duración mínima de uno o dos meses consecutivos cada dos años, que afecta al menos a una de las estaciones en el área de estudio. Esto indica que los procesos biológicos, muchos de 
los cuales ocurren en días o semanas, son muy vulnerables en el área de estudio.

Figura 4. Transcurso del SPI mensual en la estación Yaritagua. (A. periodo 1980-1999; B. periodo 20002014).
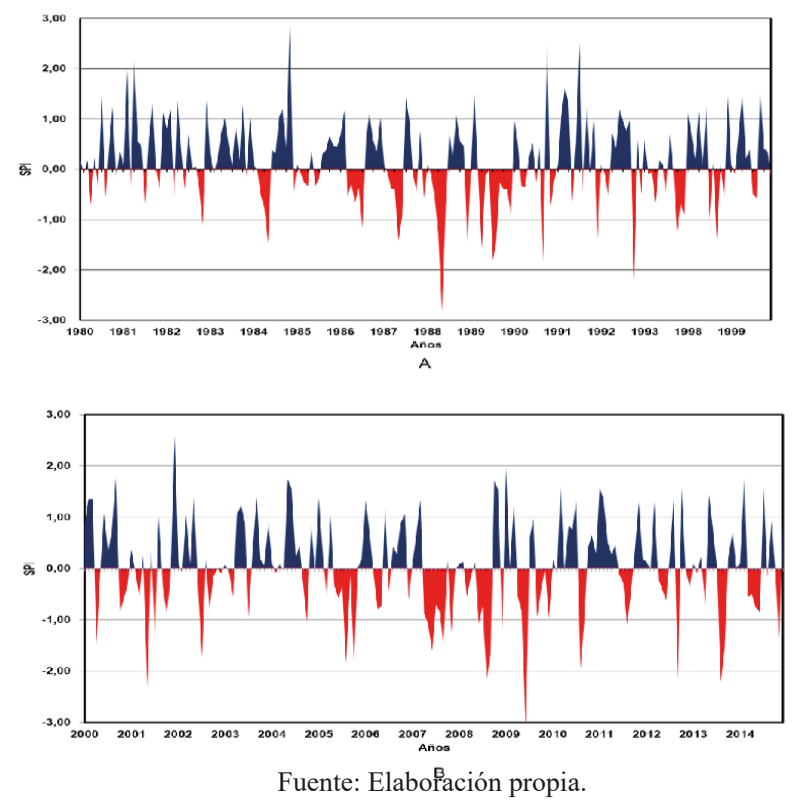

Figura 5. Transcurso del SPI mensual para el periodo (2004-2014) en la estación Las Cuibas.

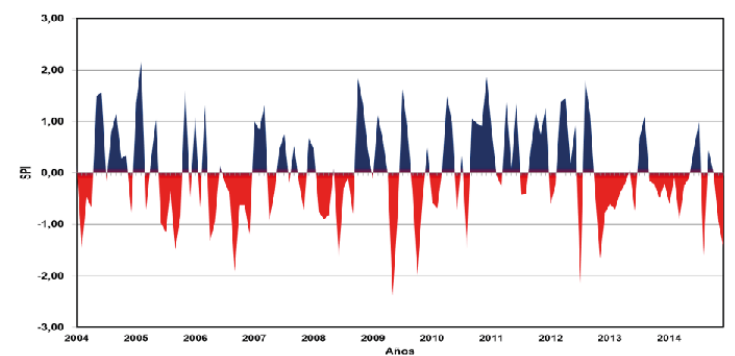

Fuente: Elaboración propia.

Según Lapinel y Báez (2007) a propósito del nivel de agrupamiento presentado en esta investigación, indican que la intensidad del SPI mensual refleja la condición de sequía inmediata, por lo que es un indicativo de la humedad en el suelo y el stress vegetativo, lo cual permitiría establecer ciertos impactos y medidas preventivas en comunidades rurales y agrícolas. A raíz de esto, Hare y Ogallo (1993) reseñan que los procesos atmosféricos que conducen a la sequía son muy complejos, destacando la escasez de humedad atmosférica, la insuficiencia de sistemas generadores de lluvia, la persistencia de una fuerte subsidencia o una combinación de alguno de los factores mencionados.

\section{Magnitud de la sequía}

Para describir la gravedad de los eventos de sequía según el SPI mensual, se calculó la magnitud de cada una de las sequías detectadas; clasificadas en cinco clases señaladas. Por consiguiente, se presentan los gráficos que reflejan su frecuencia de ocurrencia, en otras palabras, para cada clase de magnitud se grafica el riesgo de que se presente una sequía de esa magnitud.

Seguidamente para el grupo de estaciones, predominan las sequías fuertes en la estación El Cují (figura 6a); Por su parte, para la estación Quíbor predominan las sequías poco fuertes y extremadamente fuertes (figura 6b), ambas categorías con el 29,40\%. Entre tanto, la estación Yaritagua presenta el predominio de los eventos de sequías fuertes con un 29,0\% (figura 6c).

Particularmente, para la estación Las Cuibas se observan en un $54,5 \%$ los eventos de sequía extremadamente fuertes (figura 6d). 
Figura 6. Frecuencia de ocurrencia de eventos de sequía según las clases de magnitudes (SPI mensual). (A. El Cují; B. Quíbor; C. Yaritagua; D: Las Cuibas). Fuente: Elaboración propia.

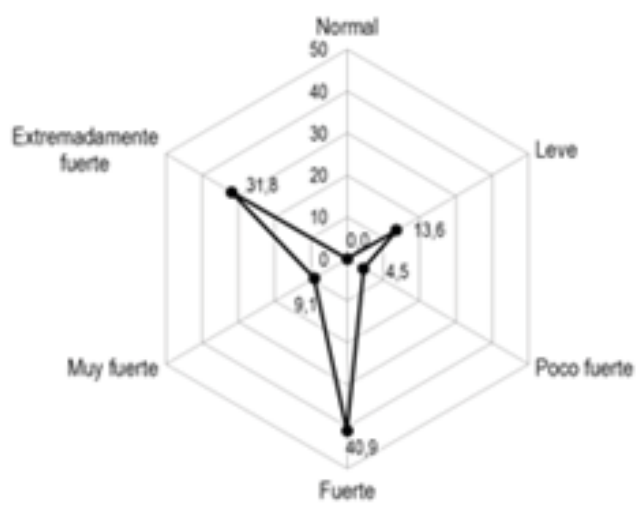

- Frecuencia de ocumencia (9) en estacion El Cuj

A

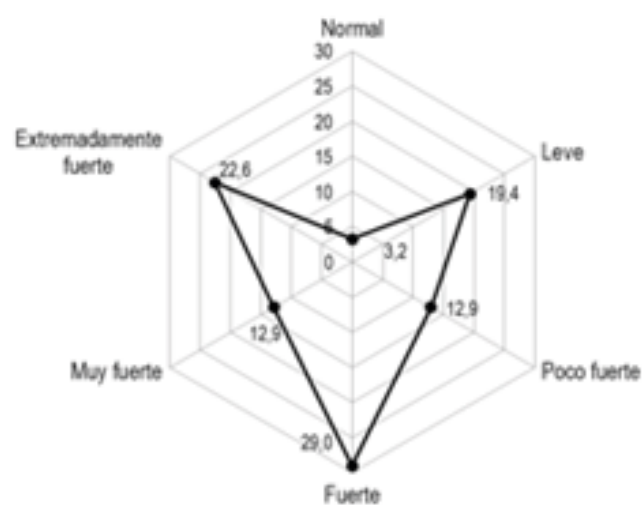

C

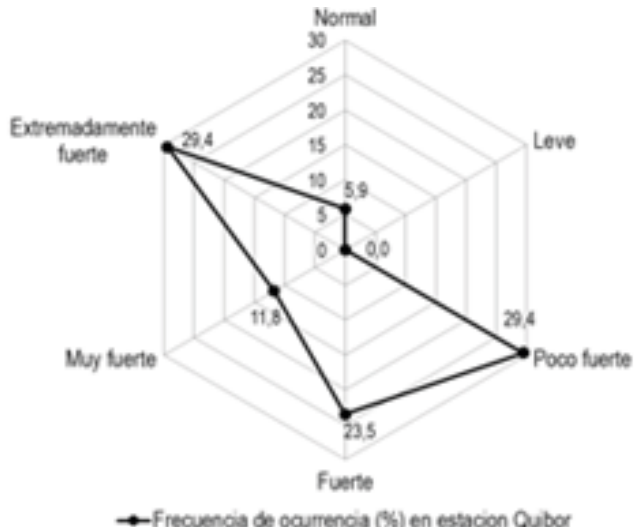

B

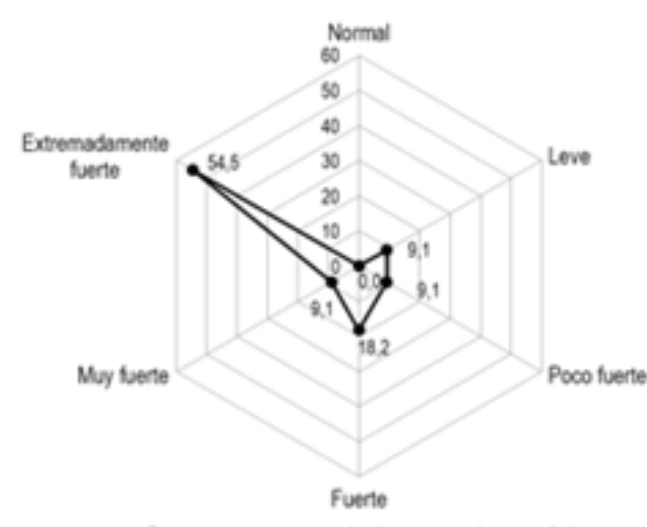

- Frecuencia de coumencia (\%) en estacion Las Cubas

D 
En lo concerniente a este comportamiento de la sequía, el documento emitido por Centella et al. (2007) indica que existe una alta probabilidad de que en los graves procesos de sequía más recientes también esté presente el componente antrópico, debido a que existen señales perceptibles de que el hombre es en parte responsable de las alteraciones observadas en el clima en las últimas décadas. Por ello, la sequía como fenómeno propio de la variabilidad climática natural, debe ser evaluada en el contexto de la superposición de señales climáticas de baja y alta frecuencia (multidecadales, decadales, multianuales), las cuales responden a ajustes del sistema climático.

A título ilustrativo, la distribución de las magnitudes de las sequías en las estaciones Yaritagua y Quíbor se representan en la figura 7, siendo la estación de Quíbor la que mayor número de magnitudes extremadamente fuertes (5 años). El intenso evento de sequía meteorológica iniciado en la época lluviosa del 2009, se prolongó por unos dos meses consecutivos, generando un déficit altamente significativo en todo el país, particularmente en la región del sistema coriano, la cual ha soportado los impactos de continuos déficit en los acumulados mensuales desde los últimos 10 años.

Figura 7. Distribución de las magnitudes de las sequías durante el periodo (2000-2014) para las estaciones Yaritagua y Quíbor. Fuente: Elaboración propia.

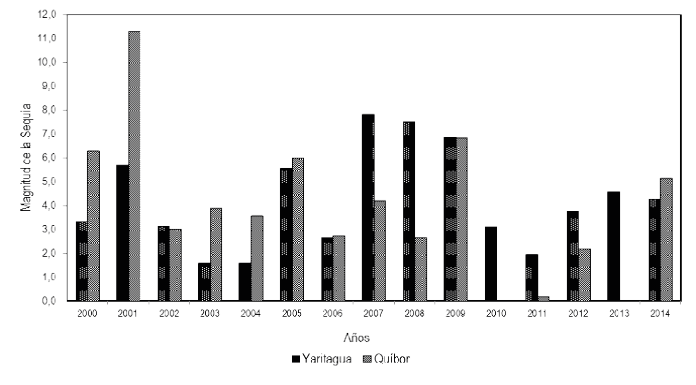

\section{Duración de las magnitudes máximas de las sequías}

Los resultados discutidos evidenciaron que en algunas estaciones bajo estudio se han presentado ciertos eventos de magnitud extremadamente fuertes; sin embargo, estos episodios han sido localizados.

Para estas estaciones bajo estudio (tabla 5) se resalta la máxima magnitud de Quíbor $(11,29)$, la cual tuvo una duración de cinco meses consecutivos durante el 2001, generando uno de los años más secos del periodo histórico evaluado en esa localidad. Durante el periodo 2001-2002, en la cordillera central también se registró un evento de sequía prolongado que afectó a los embalses de gran importancia en esta zona del país.

Tabla 5. Duración de las magnitudes máximas detectadas en las estaciones bajo estudio (Grupo II).

\begin{tabular}{ccccc}
\hline \multirow{2}{*}{ Estación } & Magnitud & \multicolumn{2}{c}{ Duración } & $\begin{array}{c}\text { Lluvia total anual } \\
(\mathrm{mm})\end{array}$ \\
\cline { 2 - 4 } & & Periodo & Año & 606,1 \\
El Cuji & 11,18 & Jun-Jul/Sep-Oct & 2005 & 407,8 \\
& 7,5 & May-Jun/Oct/Dic & 2003 & 341,7 \\
& 7,49 & Abr/Jul-Ago/Dic & 2013 & 468,5 \\
\hline \multirow{5}{*}{ Quíbor } & 11,29 & Mar-Abr/Jun/Sep & 2008 & 177,7 \\
& 6,83 & Abr-Ago/Nov & 2001 & 376,4 \\
& 6,28 & May-Jun & 2009 & 466,3 \\
\hline \multirow{4}{*}{ Yaritagua } & 7,81 & Abr/Ago/Oct & 2000 & 600,6 \\
& 7,5 & Juy-Jun/Sep/Nov & 2007 & 789 \\
\hline \multirow{3}{*}{ Las Cuibas } & 7,01 & Abr/Jul-Ago & 2008 & 616,7 \\
& 7,93 & Abr/Sep/Dic & 2006 & 682,9 \\
& 6,25 & May/Oct & 2009 & 721,6 \\
\hline
\end{tabular}

Fuente: Elaboración propia.

Al cierre del periodo seco noviembre de 2000 a abril de 2001, importantes déficits en los acumulados mensuales de la lluvia se produjeron en la mayoría de las estaciones, con amplias zonas donde los acumulados de las lluvias estuvieron por debajo de la normal. Este 
suceso persistió durante los meses del periodo lluvioso subsiguiente (mayo-septiembre 2001) en estas mismas zonas, ampliando su extensión en casi todo el territorio nacional.

\section{Discusión de resultados}

De acuerdo con los aportes de Hernández (2015) se indica que la sequía que se inició en el 2013, continuó durante gran parte de la temporada lluviosa 2014. De septiembre a octubre de 2014 hubo un ligero incremento en las precipitaciones que contribuyó a la disminución del déficit en algunas regiones llevándolo del rango extremadamente seco al cercano a lo normal, en noviembre en casi todo el país y posteriormente en diciembre de 2014 disminuyó considerablemente la sequía, a excepción de la zona costera oriental e insular (Cumaná, Guiria, Porlamar), el Vigía en Mérida, Kavanayen en la Cuenca del Caroní del estado Bolívar.

El estudio anterior señala que en septiembre de 2015 las precipitaciones registradas fueron originadas por el pasaje de las ondas tropicales, y en segundo orden, por una ligera actividad de la Zona de Convergencia Intertropical (ZCIT), sin embargo, las lluvias no alcanzaron valores significativos, ubicándose en gran parte del territorio nacional por debajo de los promedios históricos registrados, lo que provocó anomalías negativas de precipitaciones; es decir, déficit acumulado de precipitación desde abril a septiembre de 2015, que produjeron sequías y afectaron los niveles de algunos embalses, citándose: la región occidental, con los embalses Tres Ríos, Manuelote y Tulé del estado de Zulia, Barrancas y Mapará en el estado Falcón; en la región central el embalse Canoabo del estado Carabobo, los embalses Guárico y Tierra Blanca del estado Guárico.

En general, las tierras para el desarrollo agrícola en la mayor parte del estado Lara, presentan problemas en su constitución física, química y biológica, lo que ha generado la disminución gradual de la productividad por el mal manejo, así mismo, se resalta el hecho de que una significativa proporción de productores agrícolas buscan solventar esta situación con el aumento de superficie incorporando nuevas tierras. De acuerdo a los aportes del Programa de acción nacional de lucha contra la desertificación y mitigación de la sequía (Marn, 2004) a toda esta realidad se une la reducida capacidad de recuperación de la cobertura vegetal, debido a la agresividad climática y limitaciones edáficas ante la extracción de materias primas forestales y el pastoreo extensivo.

De lo expuesto, vale la pena señalar que entre las causas más destacadas que se atribuyen al origen de estos problemas de desertificación y sequía, destacan la pobreza, la falta de educación ambiental, la poca presencia de organismos oficiales, la deficiente política para el desarrollo de estas áreas, la sobreexplotación de los acuíferos, la aridez y poca fertilidad de los suelos, las pocas alternativas de subsistencia y la carencia y/o difícil acceso a créditos para su desarrollo; además de los procesos naturales de erosión. Es precisamente por esto, que se requiere de la implementación de medidas e incentivos que mitiguen o frenen los daños ecológicos, en alternancia con el aprovechamiento sostenido, organizado y capacitado.

\section{Conclusiones}

Entre las principales causas de la sequía meteorológica en la región natural denominada Sistema Coriano está la variabilidad natural del clima, aunque se acepta que su origen es el resultado de una compleja interacción entre factores de tipo natural representados por las alteraciones en los patrones de circulación atmosférica, fenómenos como El Niño 
principalmente, y factores antropogénicos en la región, tales como, la quema de combustibles fósiles, deforestación, cambios en el uso del suelo, entre otros problemas fuertemente vinculados con el desarrollo de las actividades agrícolas en estas zonas áridas, semiáridas y subhúmedas de Venezuela.

La vulnerabilidad a la sequía es mayor en las localidades avaluadas debido a que poseen una estructura económica fuertemente volcada hacia los sectores primarios extractivos y agropecuarios, que dependen de un adecuado balance del recurso hidrometeorológico para su desarrollo. Las consecuencias económicas y sociales más severas de estos eventos en el territorio, las sufren las comunidades rurales y los pequeños y medianos productores, cuya capacidad de recuperación sin asistencia es limitada.

El SPI pudo indicar que tan vulnerables fueron las localidades evaluadas ante la sequía meteorológica, partiendo de la característica de que este índice usa como única variable la lluvia, y que permitió identificar y describir convenientemente el fenómeno, precisando su inicio, intensidad, duración y magnitud. El valor singular de esta investigación y sus resultados se encuentra en la replicabilidad y la utilidad de las recomendaciones planteadas para otras áreas de Venezuela.

\section{Referencias bibliográficas}

Ablan, M, Andressen, R, Vargas, M. P. y Acevedo, M. (2008). Propuesta metodológica para el control de calidad de datos de precipitación. Agronomía Trop, 58 (1), 57-60.

Campos, D. (2005). Agroclimatología cuantitativa de cultivos. Editorial Trillas. México.
Centella, A, Lapinel, B, Solano, O., Vásquez, R., Fonseca, et al. (2007). La sequía meteorológica y agrícola en la República de Cuba y la República Dominicana. Programa de Desarrollo de las Naciones Unidas.

Colotti, E, Cedeño, M, y Montañez, C. (2013). La sequía meteorológica y la variación de la superficie agrícola en la Isla de Margarita, estado Nueva Esparta, Venezuela período 1972-2004. Terra Nueva Etapa, XXIX (45), 11-53.

Edwards, D. C, McKee, T.B. (1997). Characteristics of 20th Century drought in the United States at multiple time scales. Atmospheric Science Paper No. 634. Climatology Report (97-2), USA: Colorado State University, Fort. Collins.

Guevara, E, y Paredes, F. (2007). Influencia de variables macroclimáticas sobre el régimen pluviométrico en el estado Cojedes, Venezuela. Revista Ingeniería $U C, 14$ (3), 49-56.

Hare, F. K, y Ogallo, J. A. (1993). Climate variations, drought and desertification. WMO No. 653, Ginebra.

Hernández, R. (2008). Caracterización de la sequía meteorológica en los climas Árido, Semiárido y Subhúmedo seco en los Llanos Centro Orientales de Venezuela, para el manejo de los recursos hídricos. Centro de Investigación y Postgrado. UNEFA-CIP. Universidad Nacional Experimental Politécnica de la Fuerza Armada, Maracay, Venezuela. 
Hernández, R. (2015). Caracterización espacial de la sequía meteorológica (SPI) a nivel semestral noviembre 2014 hasta septiembre 2015, para el territorio nacional. Baruta: Inameh.

Ibarra, E, y Mendoza, N. (2004). Caracterización de la sequía meteorológica en la Cuenca Alta del Río Guárico. En: VII Congreso Venezolano de Ingeniería Agrícola. Octubre, 2004. Maracay, Venezuela.

Lapinel, B, y Báez, R. (2007). Sistema de diagnóstico y vigilancia regional de la sequía. En: Planos-Gutiérrez E, Fernández-Cueto L, Meerhoff , E. (Eds.) UNESCO-PHI Memorias de la $V$ Conferencia Mundial Proyecto Friend Amigo para América Latina y el Caribe. La Habana, Cuba. Recuperado de: $\quad$ http://unesdoc.unesco.org/ images/0015/001567/156791s.pdf

Maracara, L. (2007). Evaluación del efecto de la sequía meteorológica sobre el caudal medio del Río Tuy para un periodo de tiempo de 25 años (1951-1975). Facultad de Agronomía, Universidad Central de Venezuela. Maracay, Venezuela.

Mckee, T, Doesken, N., y Kleist, J. (1993). The relationship of drought frequency and duration to time scales. En: Proceedings of the Eighth Conference on Applied Climatology, 17-22 January 1993, Anaheim, California, USA. American Meteorological Society.

Mendoza M. y Puche M. (2007). Evaluación de la ocurrencia de sequía en localidades de Venezuela. Rev. Fac. Agron, 24 (4), 661-678.
Mendoza N, Méndez M. y Lobo D. (2007). Influencia de la capacidad de almacenamiento del suelo en la evaluación de la sequía agrícola. En: VII Congreso Venezolano de Ingeniería Agrícola. Octubre 2007. Maracay, Venezuela.

Ministerio de Ambiente y de los Recursos Naturales. Marn. (2004). Programa de acción nacional de lucha contra la desertificación y mitigación de la sequía delaRepúblicaBolivarianadeVenezuela. Caracas: Editorial Fundambiente. 106p. Recuperado de http://www.unccd.int/ ActionProgrammes/venezuela-spa2004. pdf

Ministerio del Ambiente y de los Recursos Naturales. Marn. (2005). Primera Comunicación Nacional en Cambio Climático de Venezuela. Caracas: Programa de las Naciones Unidas para el Desarrollo (PNUD), Fondo Mundial para el Medio Ambiente $\mathrm{y}$ Fundambiente.

Núñez, L. D. (2013). Variabilidad de la sequía meteorológica de largo plazo en la parte mexicana de la cuenca del Río Bravo. Universidad Autónoma de Nuevo León, Facultad De Ciencias Forestales, México. Recuperado de: http://cdigital. dgb.uanl.mx/te/1080253510.pdf

Olivares, B, Cortez, A., Parra, R., Rodríguez, M., y Guevara, E. (2013). Aplicación de procedimientos estadísticos para el control de calidad de las series de precipitación mensual de los llanos orientales venezolanos. Revista de la Facultad de Agronomía, 30 (3): 367-391. 
Ortega-Gaucin, D, y Velasco, I. (2013). Aspectos socioeconómicos $\mathrm{y}$ ambientales de las sequías en México. Aqua-LAC, 5 (2), 78 - 90.

Paredes, F, Rumbo, L, Guevara, E., y Carballo, N. (2006). Caracterización histórica de las sequías extremas del Río Tirgua en el estado Cojedes. Revista de Ciencia y Tecnología Agrollanía, 3, 101-113.

Paredes, F, Millano, J. L, y Guevara, E. (2008). Análisis espacial de las sequías meteorológicas en la región de Los Llanos de Venezuela durante el período 1961-1996. Revista de Climatología, 8, $15-27$.

Paredes, F, y Guevara, E. (2010). Desarrollo y evaluación de un modelo para predecir sequías meteorológicas en Los Llanos de Venezuela. Revista Bioagro, 22 (1), 3-10.

Parra, R, y Cortez, A. (2005). Control de calidad de series de precipitación de las series de precipitación del INIA Venezuela en el periodo 1970-2000. Rev. Arg. de Agrometeorología, (5-6): 63-73.

Thom, H. (1971). Some methods of climatological analysis. Nota técnica No 81, OMM No 199, TP 103, Secretaría de la OMM, Ginebra, Suiza, pp. 1-11.

Velasco, I, Ochoa, L., y Gutiérrez, C. (2005). Sequía, un problema de perspectiva y gestión. Revista Región y Sociedad, XVII (34), 35-71.

Wilhite, D.A. (2000). Drought as a natural hazard.En: Wilhite, D.A.(Ed.), Drought: A Global Assessment (pp:3-18). London, UK: Routledge Publishers.
Wu H., Hayes M.L., Wilhite D.A., Svoboda, M.D. (2005). The effect of the length of record on the Standardized Precipitation Index calculation, Int. J. Climato, 25, 505-520. 